\title{
FORUM
}

\section{Die neuen sicherheitspolitischen Herausforderungen und die internationale Rolle Europas}

\author{
Klaus Scharioth*
}

Einführend einige Anmerkungen zu den grundlegenden Parametern der internationalen Sicherheitspolitik, die die Gemeinsame Außen- und Sicherheitspolitik (GASP) und die Europäische Sicherheits- und Verteidigungspolitik (ESVP) beeinflussen.

- Globalisierung: Die transnationale Vernetzung politischer, wirtschaftlicher und sozialer Akteure ist ein seit langem bekanntes Phänomen. Ausmaß und Qualität der transnationalen Interaktion haben sich jedoch in den vergangenen 10 bis 15 Jahren derart dramatisch intensiviert, dass die Globalisierung heute eine entscheidende Determinante der internationalen Beziehungen darstellt.

- Neue Bedrohungen: Terrorismus, die Verbreitung von Massenvernichtungswaffen, regionale Konflikte, das Scheitern von Staaten und die organisierte Kriminalität stellen heute die Hauptbedrohungen dar.

- Das internationale System: Der Kalte Krieg und die Teilung Europas gehören glücklicherweise der Vergangenheit an. Auch im internationalen System von heute, das durch eine große Zahl an Staaten und nichtstaatlichen Akteuren gekennzeichnet ist, spielen die Vereinigten Staaten eine besonders herausragende Rolle. Ohne ihren Beitrag sind die großen sicherheitspolitischen Herausforderungen unserer Zeit nicht zu lösen.

- Die strategische Dimension der Europäischen Union: Vor dem Hintergrund veränderter sicherheitspolitischer Parameter hat sich auch die Rolle der Europäischen Union verändert. Da wir die strategische Rolle nicht länger allein den Vereinigten Staaten überlassen und uns zurücklehnen können, wurde die strategische Ausrichtung der Union um eine neue sicherheitspolitische Dimension erweitert. Die Herausforderungen, denen wir gegenüberstehen, lassen der Europäische Union keine andere Wahl, als aktiv zu Frieden und Sicherheit in der Welt beizutragen.

\section{ESVP - Grundlagen und jüngere Entwicklungen}

Die ,Geburtsstunde“ dieser neuen Europäischen Sicherheits- und Verteidigungspolitik (ESVP) schlug im Juni 1999 unter deutscher Präsidentschaft in Köln. Die Schlussfolgerungen des Europäischen Rats von Helsinki aus dem Jahre 1999 bringen die Logik der ESVP in wenigen Sätzen auf den Punkt: „Der Europäische Rat unterstreicht seine Entschlossenheit, die Union in die Lage zu versetzen, autonom Beschlüsse zu fassen und in den Fällen, in denen die NATO als Ganzes nicht beteiligt ist, als Reaktion auf internationale Krisen EU-geführte militärische Operationen einzuleiten und durchzuführen. Dabei ist unnötige Duplizierung zu vermeiden. Dieser Prozess impliziert nicht die Schaffung einer europäischen

* Dr. Klaus Scharioth, Staatssekretär des Auswärtigen Amts, Berlin. Der Beitrag basiert auf einem Vortrag des Autors (Originaltext Englisch) im Rahmen der vom IEP durchgeführten Konferenz, Young Faces ‘ am 20. Januar 2005 in Berlin. Die Konferenz fand zur Eröffnung des gemeinsamen Programms ,European Foreign and Security Policy Studies“ der VolkswagenStiftung, der Compagnia di San Paolo, Turin/Italien, und des Riksbankens Jubileumsfond, Stockholm/Schweden statt. 
Armee. “1 Gleichzeitig beschlossen die Staats- und Regierungschefs, ,die Ressourcen im Bereich der zivilen Krisenbewältigung, in dem die Union und die Mitgliedstaaten bereits über beträchtliche Erfahrungen verfügen, [zu] verbessern und effizienter [zu] nutzen.“ ${ }^{\text {2 }}$

In den vier ersten Jahren der ESVP entwickelte die Union operative militärische und zivile Fähigkeiten im Krisenmanagement. Im Jahr 2003 wurden insgesamt vier Krisenmanagement-Operationen eingeleitet. Im vergangenen Jahr kam eine weitere zivile ESVP-Operation dazu, die Rechtsstaatsmission in Georgien. Der Rat erörterte mögliche zivile ESVPMissionen mit Blick auf den Kongo, Sudan und andere Schauplätze. Auf Grundlage der Entscheidungen von NATO und Europäischer Union folgte in Bosnien-Herzegowina Ende vergangenen Jahres die Operation Althea der NATO-geführten SFOR nach.

Vor dem Hintergrund einer wachsenden Nachfrage nach internationalem Krisenmanagement trägt die Union nun einen immer größeren Teil der Last. Im Vergleich zur Situation vor 1999 ist dies eine wichtige Errungenschaft.

Die strategische Partnerschaft zwischen Europäischer Union und NATO hat dazu entscheidend beigetragen. Diese Partnerschaft bleibt eine unverzichtbare Voraussetzung zur Bewältigung der neuen Herausforderungen. Sie steht auf der soliden Grundlage von Berlin Plus: Dauervereinbarungen, die der Europäischen Union den Zugriff auf Mittel und Fähigkeiten der NATO ermöglichen. Zudem ist zu berücksichtigen, dass die 19 Staaten, die gleichzeitig Mitglieder der Europäischen Union wie der NATO sind, ihren Beitrag zu beiden Organisationen jeweils aus einem einheitlichen, nationalen Streitkräfte-Pool beisteuern. Es entspricht dieser Logik, dass sich etwa auch die schnell verlegbaren Gefechtsverbände der Europäischen Union (,Battle Groups') und die NATO Reaktionskräfte (NATO Response Force, NRF) gegenseitig verstärken.

Die Verbesserung der EU-Handlungsfähigkeit durch die ESVP wurde zu einem dynamischen Lernprozess. In Berlin hätten beispielsweise nur wenige vorhergesehen, dass die Union innerhalb von drei Monaten nach dem Start ihrer allerersten militärischen Operation in Mazedonien Anfang 2003 eine weitere Militäroperation in Afrika - dieses Mal ohne Rückgriff auf Mittel und Fähigkeiten der NATO - beginnen würde. Genau dies aber geschah: Deutschland stellte seine anfänglichen Vorbehalte zurück und die Operation Artemis in der Demokratischen Republik Kongo wurde ein in jeder Hinsicht erheblicher Erfolg. Artemis bewies, dass die Union in der Lage ist, bestimmte Arten autonomer Operationen kurzfristig durchzuführen und Truppen in ein schwieriges Umfeld zu verlegen. Artemis war der Beleg, dass die ESVP in Afrika sehr wohl eine Rolle einnehmen konnte und führte zur Entwicklung des Gefechtsverbands-Konzepts, das Frankreich, das Vereinigte Königreich und Deutschland vor einem Jahr vorgeschlagen haben. Artemis beeinflusste auch die Diskussion darüber, welche kollektiven Fähigkeiten die Europäische Union für autonome Operationen benötigt, und führte so zu der Entscheidung, im Militärstab der Union in Brüssel eine zivilmilitärische Zelle zu schaffen. Diese Zelle wird unter anderem in der Lage sein, ein Operationszentrum zur Planung und Führung von Operationen einzurichten.

Ein weiteres Beispiel dafür, wie schnell sich die ESVP entwickelt hat, ist die Europäische Verteidigungsagentur. Jahrzehntelang wurde über das Konzept einer Rüstungsagentur in Europa diskutiert. Vor zwei Jahren schlugen Deutschland und Frankreich im Konvent dann die Schaffung einer Agentur vor. Der Vorschlag war Teil des Entwurfs eines Verfassungsvertrags. Seit vergangenem Sommer hat die Agentur ihre Arbeit aufgenommen. Sie wird

1 Europäischer Rat (Helsinki): Schlussfolgerungen des Vorsitzes, 10./11.12.1999, abrufbar unter http://ue.eu.int/ ueDocs/cms_Data/docs/pressData/de/ec/00300-r1.d9.htm (letzter Zugriff: 8. 3. 2005).

2 Europäischer Rat (Helsinki): Schlussfolgerungen des Vorsitzes, Anlage IV. 
eine wichtige Rolle bei der Entwicklung der Fähigkeiten spielen und uns hoffentlich helfen, die noch bestehenden Defizite zu beseitigen, die der Handlungsfähigkeit der Union derzeit noch deutliche Grenzen setzen.

\section{Ziviles Krisenmanagement}

Häufig konzentrieren sich Diskussionen zur ESVP auf die militärischen Aspekte. Von Anbeginn der ESVP hat Deutschland jedoch Wert darauf gelegt, dass zivile und militärische Mittel gleichberechtigt entwickelt werden müssen. Die Erfahrungen auf dem Balkan und in Afghanistan zeigen, dass nur eine Kombination aus zivilen und militärischen Instrumenten zum Erfolg führt. Gerade dieser umfassende Ansatz ist ein Markenzeichen, das die ESVP deutlich von anderen Konzepten unterscheidet. Allerdings hinkt das zivile Krisenmanagement noch deutlich hinter dem militärischen her. Gegenwärtig wäre die Union nicht in der Lage, kurzfristig , anspruchsvolle‘ zivile Missionen größeren Maßstabes in Krisengebiete zu verlegen. Die zivilen Fähigkeiten der Mitgliedstaaten können in aller Regel nicht ad-hoc für ESVP-Operationen abgerufen werden und sind daher nicht schnell einsetzbar. Daher ist ein langfristiger, weitsichtiger Ansatz erforderlich. Aus diesem Grund schlugen Deutschland und andere Partner ein „Konsolidiertes Ziviles Planziel“ vor, das der Europäische Rat im Dezember 2004 beschlossen hat. Dieses Planziel soll strategische Parameter für das zivile Krisenmanagement definieren, die zu einer systematischeren Entwicklung ziviler Fähigkeiten führen. Dieser Ansatz wird in Anlehnung an die Entwicklung der militärischen Fähigkeiten der ESVP implementiert.

\section{Die Europäische Sicherheitsstrategie}

Ein zweites Beispiel für die neue strategische Dimension der Europäischen Union ist die Europäische Sicherheitsstrategie ${ }^{3}$ (ESS). Klarheit über politische Ziele und die Mittel, diese zu erreichen, sind eine Voraussetzung für eine kohärente Sicherheitspolitik. Aus diesem Grund stellt die Sicherheitsstrategie vom Dezember 2003 einen Meilenstein in der Entwicklung der Sicherheitspolitik der Europäischen Union dar.

Die Strategie konzentriert sich auf drei strategische Ziele, die die Sicherheit der EU-Bürger gewährleisten und Stabilität über die Europäische Union hinaus fördern sollen. Angesichts der neuen Bedrohungen muss sich die Union erstens frühzeitig und mit dem gesamten ihr zur Verfügung stehenden Instrumentarium engagieren. Die erste ,Verteidigungslinie ' wird hierbei oft im Ausland liegen. Wann immer möglich, soll bereits gehandelt werden, bevor sich die Krise entwickelt. Zweitens legt die ESS einen Schwerpunkt auf die Schaffung von Frieden und Stabilität in der unmittelbaren Nachbarschaft der Union. Dies bezieht sich auf die langjährige Erfahrung der Union mit Stabilisierungsprozessen in ihrer Umgebung. Ziel ist, dass von den östlichen Grenzen der Europäischen Union bis zum Mittelmeer ein Bogen verantwortungsvoll regierter Staaten entsteht. Und schließlich betont die Strategie drittens die Wichtigkeit des Völkerrechts sowie die Rolle der Vereinten Nationen. Hierfür haben wir den Begriff ,effektiver Multilateralismus' geprägt.

Doch die Sicherheitsstrategie bleibt nicht bei der Beschreibung der strategischen Ziele stehen. Sie beschreibt auch die Konsequenzen, die diese Ziele für die EU-Politik in der Praxis haben. Sie plädiert für eine aktivere Außenpolitik mit dem gezielten Einsatz des breiten Spektrums von diplomatischen, handels- und entwicklungspolitischen Instrumenten, ein-

3 Vgl. Rat der Europäischen Union: Ein Sicheres Europa in einer besseren Welt: Europäische Sicherheitsstrategie, abrufbar unter http://ue.eu.int/uedocs/cms_data/docs/2004/4/29/European\%20Security\%20Strategy.pdf (letzter Zugriff: 20.5.2005). 
schließlich des zivilen und militärischen Krisenmanagements. Zu Recht unterstreicht sie die Wichtigkeit der Zusammenarbeit mit strategischen Partnern und erwähnt die Vereinigten Staaten, Russland, Japan, China, Kanada und Indien in diesem Zusammenhang. Der strategische Dialog zwischen beiden Seiten des Atlantiks ist entscheidend. Er wurde nach dem 11. September und vor dem Irakkrieg schmerzlich vermisst. Wir brauchen einen umfassenden Dialog, der keine kontroversen Themen scheut. Wir müssen auf ein gemeinsames Verständnis von Nicht-Weiterverbreitung, Terrorismus, Bedrohungsanalyse hinarbeiten. Dies muss aber auch die Prinzipien der Anwendung militärischer Gewalt einschließen. Mit der ESS hat die Europäische Union - zum ersten Mal in ihrer Geschichte - einen umfassenden Rahmen für ihre Sicherheitspolitik entworfen. Die ESS selbst hat damit bereits einen wesentlichen Beitrag zur Kohärenz der GASP geleistet.

\section{Stabilisierung, Integration und, Soft Power “}

Kritische Beobachter beschrieben die Europäische Union bisher gern als wirtschaftlichen Riesen und politischen Zwerg. Wie bereits erwähnt, haben die jüngeren Entwicklungen in der GASP und in der ESVP die Europäische Union jedoch in einen weitaus glaubwürdigeren Akteur auf dem Gebiet der Diplomatie und der Sicherheitspolitik verwandelt. Zugleich wäre es aber falsch, die Wichtigkeit der wirtschaftlichen Dimension der Union für unsere Sicherheit zu unterschätzen. Ein Großteil des weltweiten Beitrags der Europäischen Union zu Frieden und Sicherheit war und ist in der Tat wirtschaftlicher Natur. Grundlage dieses Beitrags sind Handelspolitik, Entwicklungshilfe und humanitäre Hilfe. Seit vielen Jahren verfolgt die Europäische Union die Politik, Frieden und Stabilität in ihrer Nachbarschaft über einen dualen Prozess zu fördern: durch die Stabilisierung der Nachbarregionen durch regionale Zusammenarbeit und Partnerschaften einerseits; und durch die volle EU-Mitgliedschaft andererseits. Die Erweiterung war und ist wahrscheinlich die effektivste Sicherheitspolitik und Krisenprävention der Union. Auch wenn die Erweiterung ihre Grenzen hat, so entwickeln auch Staaten, die nicht Mitglieder der Union werden, dennoch sehr enge Beziehungen zur Union. Eine ganze Region von Osteuropa bis zum Mittelmeer wird so immer stärker durch gemeinsame Werte, offene Märkte und mannigfache Zusammenarbeit in verschiedensten Bereichen zusammen gehalten.

,Soft power' ist ein weiterer Aspekt, der keinesfalls unterschätzt werden sollte. In vielen Teilen der Welt wird die Europäische Union als ein Modell für friedliche Zusammenarbeit und politische und wirtschaftliche Entwicklung betrachtet. Im Zusammenhang mit unserem Engagement für Multilateralismus und Völkerrecht verleiht uns dies Glaubwürdigkeit, wenn es um die Förderung von Stabilität und Demokratie im Ausland geht.

\section{Die Europäische Verfassung}

Der, Vertrag über eine Verfassung für Europa“ ist ein großer Schritt nach vorne, insbesondere im Bereich der Außen- und Sicherheitspolitik. Pars pro toto möchte ich nur ein wichtiges Element der Verfassung herausgreifen, das die internationale Rolle Europas wesentlich verändern wird: der Europäische Außenminister, unterstützt durch den Europäischen Auswärtigen Dienst, ist eine entscheidende neue Institution. Lassen Sie uns nicht vergessen, dass der Außenminister für den gesamten Bereich des Krisenmanagements verantwortlich sein wird. Der Militärstab der Union wird ebenso zu seinem Verantwortungsbereich gehören wie die Verteidigungsagentur. Er wird für den Bereich des zivilen Krisenmanagements im Sekretariat des Rates ebenso zuständig sein wie in der Europäischen Kommission. Javier Solana wurde bereits zum ersten designierten Außenminister ernannt. Das Zusammenführen 
aller relevanten Aufgaben in den Verantwortungsbereich einer Person ist ein institutioneller Quantensprung, der die Fähigkeit der Europäischen Union, rasch, effektiv und auf kohärentere Weise zu handeln, nachhaltig verbessern wird.

\section{Ausblick}

Die Europäische Union ist ohne jeden Zweifel eine Friedensmacht - in Europa und darüber hinaus. Aber um den Herausforderungen des 21. Jahrhunderts gewachsen zu sein, müssen wir folgenden grundlegenden Anforderungen genügen:

- Die Union hat eine strategische Dimension hinzugewonnen. Sie hat keine andere Wahl, als ihren Beitrag zu Frieden und Sicherheit in der Welt zu leisten.

- Wir müssen uns über unsere Ziele und über die Instrumente, die wir anwenden wollen, im klaren sein. Die ESS ist ein Dokument erster Güte, aber wir müssen es stets aktualisieren und in die Praxis umsetzen.

- Das Profil der Union wird zu einem großen Teil das einer ,Zivilmacht‘ bleiben. Handelsund Entwicklungspolitik etwa müssen zur Förderung von Sicherheit und Stabilität eingesetzt werden.

- Zugleich müssen unsere militärischen und zivilen Fähigkeiten im Krisenmanagement weiter verbessert werden. Die Tsunami-Katastrophe in Asien ist ein weiterer Anlass, die Fähigkeit der Europäischen Union zur schnellen Reaktion auf Krisen in einem umfassenden, auf den verschiedenen Säulen basierenden Ansatz zu untersuchen.

- Wir müssen einen reibungslosen Übergang zwischen militärischem und zivilem Krisenmanagement sicher stellen und auch die Entwicklungshilfe in den Prozess einbeziehen.

- Ungeachtet der verbesserten Fähigkeiten der Europäischen Union hängt unsere Fähigkeit, Einfluss auszuüben, auch von unseren strategischen Partnern ab. Die Vereinigten Staaten bleiben der wichtigste strategische Partner. Wir brauchen ein starkes Europa, aber wir brauchen auch eine transatlantische Partnerschaft unter gleichberechtigten Partnern.

- Die NATO bleibt das Kernstück der transatlantischen Partnerschaft in der Sicherheits- und Verteidigungspolitik.

- Außerhalb Europas wird Afrika für die ESVP eine Region von zunehmender Bedeutung werden. Wo immer es möglich sein wird, werden wir mit der Afrikanischen Union (AU) und den Vereinten Nationen (VN) zusammenarbeiten. Dort, wo AU und VN nicht selbst tätig werden können, stellen ESVP-Missionen eine Option dar.

- In diesem Zusammenhang ist und bleibt der effektive Multilateralismus - unter Betonung der besonders wichtigen Rolle des VN-Sicherheitsrates - die Grundlage der EU-Politik.

- Und schließlich dürfen wir nicht nachlassen, unseren Bürgern die Sicherheitspolitik der Union und insbesondere die ESVP zu erklären. Eine stärkere Rolle der Union in der Weltpolitik findet breite Unterstützung. Aber wir dürfen diese Unterstützung nie für selbstverständlich halten.

Wir leben in einer gefährlichen Welt. Es ist unser gemeinsames Ziel, alles dafür zu tun, dass die Europäische Union sich auch weiterhin zu einem fähigen und glaubwürdigen Akteur auf dem Gebiet der internationalen Sicherheit entwickelt. 